\title{
Flora of Singapore precursors, 12. Notes on various Rubiaceae genera
}

\author{
I.M. Turner \\ Singapore Botanical Liaison Officer, \\ Royal Botanic Gardens Kew, Richmond, \\ Surrey TW9 3AE, UK \\ i.turner@kew.org \\ Singapore Botanic Gardens, National Parks Board, \\ 1 Cluny Road, 259569 Singapore
}

\begin{abstract}
In preparation for the account of the Rubiaceae for the Flora of Singapore various notes are presented concerning the genera Discospermum Dalzell, Lasianthus Jack, Mussaenda L. and Psychotria L. Nomenclatural comments on the generic synonyms Landia Comm. ex Juss. (= Mussaenda) and Uragoga Baill. (= Psychotria) are made. Lectotypes are designated at the first or second step for 42 names, and one neotype is designated. Recent collections of Lasianthus griffithii Wight and Psychotria morindiflora Wall. ex Hook.f. from Singapore are highlighted.
\end{abstract}

Keywords. Discospermum, Landia, Lasianthus, lectotype, Mussaenda, Psychotria, Uragoga

\section{Introduction}

In revising various genera of the Rubiaceae for the Flora of Singapore a number of points of taxonomic and nomenclatural note required attention. These are dealt with here.

\section{Diplospora DC. versus Discospermum Dalzell}

Hooker (1880) reduced the genus Discopermum to a section of Diplospora. This synonymisation of the genera was generally accepted until Ali \& Robbrecht (1991) resurrected Discospermum. Arriola et al. (2018) have recently confirmed, using molecular techniques, that Diplospora and Discospermum do represent separate lineages in the coffee tribe. The only species of the Coffeeae native to Singapore has been referred to as Diplospora malaccensis Hook.f. in nearly all publications on the flora. Ali \& Robbrecht (1991) excluded this species from the Coffeeae, arguing that the orientation of the embryo radicle and details of the seed coat anatomy indicated membership of the tribe Hypobathreae. However, Diplospora malaccensis shows many similarities with Discospermum, particularly Discospermum beccarianum (King \& Gamble) S.J.Ali \& Robbr. The latter was demonstrated to be a close ally of the type species of Discospermum, D. sphaerocarpum Dalzell, by Arriola et al. (2018). 
I conclude that for the forthcoming Flora of Singapore Diplospora malaccensis is best placed in the genus Discospermum. There is already an appropriate combination available. It is necessary to select a lectotype, which is done below.

Discospermum malaccense (Hook.f.) Kuntze, Revis. Gen. P1. 1: 281 (1891). - Diplospora malaccensis Hook.f., Fl. Brit. India 3: 124 (1880). - Tricalysia malaccensis (Hook.f.) Merr., Univ. Calif. Publ. Bot. 15: 285 (1929). - TYPE: Malacca, 1845, W. Griffith s.n. (lectotype K [K000763121], designated here).

\section{Lasianthus}

The revision of Lasianthus in Malesia by Zhu et al. (2012) has largely been followed in preparing the account of the genus for the Flora of Singapore. As this leads to changes in the names of several species found in Singapore, it is useful to provide a listing here with synonymy and typification. A number of new lectotypes are designated here and in a few cases notes given on taxonomic decisions and current status in Singapore.

1. Lasianthus attenuatus Jack, Trans. Linn. Soc. London 14: 126 (1823). - Mephitidia attenuata (Jack) DC., Prodr. 4: 452 (1830). - Nonatelia attenuata (Jack) Kuntze, Revis. Gen. P1. 1: 291 (1891). - TYPE: Sumatra, Tappanooly [Tapanuli], 1819, W. Jack s.n. (lectotype L [L0000674], designated by Merrill, J. Arnold. Arbor. 33: 229 (1952)).

\section{1a. Lasianthus attenuatus var. attenuatus}

Nonatelia hispida Wall. in Roxburgh, Fl. Ind. 2: 187 (1824). - Mephitidia wallichii Wight \& Arn., Prodr. Fl. Ind. Orient. 390 (1834), nom. illeg. (superfl.). - Lasianthus wallichii Wight, Calcutta J. Nat. Hist. 6: 503 (1848), nom. illeg. (superfl.). - TYPE: Silhet, EIC 8442 (lectotype K [K000031602], designated by Zhu, Acta Phytotax. Sin. 32: 57 (1994); isolectotypes K-W [K001125525, K001125526, K001125527]).

Mephitidia attenuata Jack var. glabra Korth., Ned. Kruidk. Arch. 2: 220 (1851). - TYPE: Sumatra, Singalang, P.W. Korthals (not traced).

Lasianthus densifolius Miq., Fl. Ned. Ind. 2: 321 (1857). - Nonatelia densifolia (Miq.) Kuntze, Revis. Gen. P1. 1: 291 (1891). - TYPE: Java, Ungaran, F.W. Junghuhn s.n. (lectotype L [L0000679], designated by Zhu, Syst. \& Geogr. P1. 72 : 73 (2002)).

Lasianthus plagiophyllus Hance, J. Bot. 13: 196 (1875). - Mephitidia plagiophylla (Hance) Nakai, Trees Shrubs Japan 529 (1927). - Lasianthus wallichii subsp. plagiophyllus (Hance) C.Y.Wu \& H.Zhu, Acta Phytotax. Sin. 32: 57 (1994). - TYPE: China, Hongkong, Hong Kong Island, in devexis collium vallem Wongneichung [Wong Nai Chung] curcumventium, juxta coemeteria, horto publico praepositus, 21 
April 1874, H.F. Hance 18438 [leg. C. Ford], (lectotype K [K000763833], designated by Zhu, Acta Phytotax. Sin. 32: 57 (1994); isolectotypes BM [BM000945610], K [K000763831, K000763834]).

Lasianthus densifolius var. calycinus King, J. Asiat. Soc. Bengal, Pt. 2, Nat. Hist. 73: 122 (1904), as 'calycina'. - TYPE: Singapore, Chan Chu Kang, 1892, H.N. Ridley 5679 (lectotype SING [SING0030305], designated here; isolectotype CAL [CAL0000031880]).

Lasianthus densifolius Miq. var. latifolius King, J. Asiat. Soc. Bengal, Pt. 2, Nat. Hist. 73: 122 (1904), as 'latifolia'. - TYPE: [Peninsular Malaysia], Johore, Panchur, Johore River, 14 October 1900, H.N. Ridley 10948 (lectotype SING [SING0059263], designated here; isolectotype K [K001129550]).

Mephitidia tonkinensis Drake, J. Bot. (Morot) 9: 240 (1895). - Lasianthus tonkinensis (Drake) Pit. in Lecomte, Fl. Indo-Chine 3: 376 (1924). - TYPE: Tonkin, forêts du mont Bavi, 24 November 1887, B. Balansa 2656 (holotype P [P02285231]; isotype K [K000777039]).

Lasianthus brachyphyllus K.Schum. in Schumann \& Lauterbach, Fl. Schutzgeb. Südsee, Nachtr. 399 (1905). - TYPE: Papua New Guinea, Kaiser-Wilhelmsland, Sattelberg, July 1899, E.O.A. Nyman 678 (lectotype UPS [V-102855], designated here).

Lasianthus bordenii Elmer, Leafl. Philipp. Bot. 1: 11 (1906), as 'bordeni'. - TYPE: Philippines, Luzon, Bataan, Mt Mariveles, November 1904, A.D.E. Elmer 6977 (lectotype K [K000777107], designated here; isolectotypes E [E00504401], NY [NY00132080]).

Lasianthus copelandii Elmer, Leafl. Philipp. Bot. 1: 10 (1906), as 'copelandi' - TYPE: Philippines, Negros, Gimogon River, January 1905, E.B. Copeland s.n. (not traced).

Lasianthus thorelii Pit. in Lecomte, Fl. Indo-Chine 3: 389 (1924). - TYPE: Laos, Penangs, 1866-1868, Thorel 2161 (lectotype P [P02285232], first step designated by Zhu, Acta Bot. Yunan. 20: 158 (1998), second step designated here; isolectotype P [P02285233]).

Lasianthus attenuatus var. subsessilis Valeton, Bot. Jahrb. Syst. 61: 108 (1927). - TYPE: New Guinea, Jaduna, 3 April 1907, Schlechter 17334 (holotype B $\dagger$ ).

Lasianthus setosus Craib, Bull. Misc. Inform. Kew 1933: 26 (1933). - Lasianthus wallichii var. setosus (Craib) C.Y.Wu \& H.Zhu, Acta Phytotax. Sin. 32: 57 (1994). TYPE: Thailand, Ranawng [Ranong], Kao Talu, 4 February 1927, A.F.G. Kerr 11827 (lectotype K [K000777040], designated here; isolectotypes BK [BK257397], BM [BM000945596]). 
Lasianthus wallichii var. glabriusculus Deb \& M.G.Gangop., J. Bombay Nat. Hist. Soc. 84: 462, Fig. 3 (1988 ['1987']). - TYPE: Burma, 1 March 1849, H. Falconer 885 (holotype CAL; isotype CAL).

Lasianthus wallichii var. hispidocostatus H.Zhu, Acta Bot. Yunnan. 20: 158 (1998). - TYPE: China, Yunnan, Szemao, A. Henry 12789 (holotype K [K000763835]; isotype MO [MO-716813]).

In describing Nonatelia hispida, Wallich referred to specimens he had received from Silhet [Sylhet] in 1815. Given the date, these are likely to have come from M.R. Smith. Zhu effectively lectotypified the name to one of the specimens distributed by Wallich under the number 8442. One of these in the East India Company Herbarium (K-W) has an original label stating that the specimen was collected by F. De Silva in Sylhet in August 1820. It seems likely that Wallich would have seen the De Silva gatherings before the publication of the protologue in 1824, so Zhu effectively typified to an uncited specimen. If the 1815 material were to come to light, there would be grounds for making a cited specimen the lectotype (ICN (Turland et al., 2018) Art. 9.12). However, I have not traced any such material, so maintain Zhu's typification here.

1b. Lasianthus attenuatus var. minor H.Zhu, Blumea 57: 16 (2012). - TYPE: Singapore, Nee Soon, 18 March 1982, J.F. Maxwell 82-72 (holotype L [L0310278]). Lasianthus densifolius auct., non Miq., King \& Gamble, J. Asiat. Soc. Bengal, Pt. 2, Nat. Hist. 73: 122 (1904); Ridley, J. Straits Branch Roy. Asiat. Soc. 33: 99 (1900); Ridley, Fl. Malay Penins. 2: 156 (1923); Wong, Tree Fl. Malaya 4: 368 (1989); Turner, Gard. Bull. Singapore 45(1): 199 (1993); Tan et al., Gard. Bull. Singapore, Suppl. No. 3: 46, Fig. 4 (1995); Turner, Gard. Bull. Singapore 47(2): 430 (1997 ['1995']); Turner \& Chua, Checkl. Vasc. Pl. Sp. Bukit Timah Nat. Res. 67 (2011).

This has been long known as Lasianthus densifolius. Zhu made this name a synonym of Lasianthus attenuatus by lectotypification and redescribed this taxon as $L$. attenuatus var. minor. There are many points of similarity between these two taxa and they seem to overlap in form occasionally though the difference in size of the calyx lobes appears to be consistent. As I have not had the chance to examine flowering material, I maintain Zhu's system of infraspecific taxa here.

2. Lasianthus chryseus Ridl., J. Fed. Malay States Mus. 10: 143 (1920). - TYPE: Singapore, Gardens' Jungle, February 1889, H.N. Ridley 140 (lectotype K [K000763913], designated by Zhu et al., Blumea 57: 28 (2012)).

Mephitidia capitata var. ferruginea Korth., Ned. Kruidk. Arch. 2: 222 (1851). - TYPE: Borneo, Prarawin, P.W. Korthals s.n. (lectotype L [L0057527], designated here). 
Lasianthus tomentosus auct., non Blume, King \& Gamble, J. Asiat. Soc. Bengal, Pt. 2, Nat. Hist. 73: 130 (1904); Ridley, Fl. Malay Penins. 2: 161 (1923), p.p.; Wong, Tree F1. Malaya 4: 371 (1989); Keng, Concise Fl. Singapore, vol. 1, Gymn. Dicot. 157 (1990); Turner, Gard. Bull. Singapore 45(1): 200 (1993); Tan et al., Gard. Bull. Singapore, Suppl. No. 3: 46 (1995); Turner, Gard. Bull. Singapore 47(2): 433 (1997 ['1995']); Turner \& Chua, Checkl. Vasc. P1. Sp. Bukit Timah Nat. Res. 60 (2011).

This species has generally been referred to as Lasianthus tomentosus in works on the flora of Singapore.

3. Lasianthus constrictus Wight, Calcutta J. Nat. Hist. 6: 515 (1846). - Mephitidia constricta (Wight) Walp., Ann. Bot. Syst. 2: 762 (1852). - Nonatelia constricta (Wight) Kuntze, Revis. Gen. P1. 1: 291 (1891). - TYPE: Burma, Mergui, W. Griffith s.n. (holotype K [K000031672], fide Noltie, Bot. Robert Wight 414 (2005)).

Lasianthus constrictus var. latifolius Craib, Fl. Siam. 2: 209 (1934). - TYPE: Thailand, Pattani, Kao Kala Kiri, 1 April 1928, A.F.G. Kerr 14940 (lectotype K [K000777034], designated here; isolectotypes BK [BK257385], BM [BM000945603], E [E00327855], $\mathrm{K}[\mathrm{K} 000777033])$.

4. Lasianthus ellipticus Wight, Calcutta J. Nat. Hist. 6: 507 (1846). - Mephitidia elliptica (Wight) Walp., Ann. Bot. Syst. 2: 761 (1852). - Nonatelia elliptica (Wight) Kuntze, Revis. Gen. P1. 1: 291 (1891). - TYPE: [Peninsular Malaysia], Malacca, W. Griffith s.n. (holotype K [K000763923], fide Noltie, Bot. Robert Wight 415 (2005)).

Lasianthus sordidus Ridl., J. Straits Branch Roy. Asiat. Soc. 57 (1911) 60. - TYPE: [Peninsular Malaysia], Perak, Temango, July 1909, H.N. Ridley 14488 (lectotype BM [BM001190973], designated by Turner et al., Gard. Bull. Singapore 70: 356 (2018); isolectotype (K [K000763925]).

Lasianthus venulosus Ridl., J. Straits Branch Roy. Asiat. Soc. 61: 23 (1912), nom. illeg., non L. venulosus (Wight \& Arn.) Wight (1846). - TYPE: Singapore, Bukit Panjang, 1906, H.N. Ridley 12542 (lectotype K [K000763924], designated by Turner et al., Gard. Bull. Singapore 70: 357 (2018)).

Lasianthus velutinus Ridl., J. Fed. Malay States Mus. 10: 144 (1920). - TYPE: [Peninsular Malaysia], Kelantan, Chaning Woods, 4 February 1917, H.N. Ridley s.n. (lectotype SING [SING0059265], designated by Turner et al., Gard. Bull. Singapore 70: 357 (2018); isolectotype K [K000763926]).

Lasianthus scalaris Craib, Bull. Misc. Inform. Kew 1933: 26 (1933). - TYPE: Thailand, Krabi, Tambon, Kao Panom [Khao Phanom], 1 April 1930, A.F.G. Kerr 
18809 (lectotype K [K00777030, K000777029 - i.e. a single specimen over two sheets], designated here; isolectotypes BK [BK257396], BM [BM001191350]).

Lasianthus glaber auct., non Ridl., Wong, Tree Fl. Malaya 4: 373 (1989), p.p.; Turner, Gard. Bull. Singapore 45(1): 199 (1993).

Wong (1989) mistakenly reduced Lasianthus venulosus Ridl. to a synonym of $L$. glaber Ridl. This has led to an erroneous record of L. glaber in Singapore. Lasianthus glaber has much bigger stipules than L. ellipticus and Zhu et al. (2012) put it in section Stipularae.

5. Lasianthus griffithii Wight, Calcutta J. Nat. Hist. 6: 505 (1846). - Mephitidia griffithii (Wight) Walp., Ann. Bot. Syst. 2: 760 (1852). - Nonatelia griffithii (Wight) Kuntze, Revis. Gen. P1. 1: 291 (1891). - TYPE: [Peninsular Malaysia], Malacca, W. Griffith s.n. (lectotype K [K000763947], designated here).

Lasianthus spathulatus Miq., Ann. Mus. Bot. Lugduno-Batavi 4: 246 (1869). - TYPE: Borneo, 1859-1860, G.W. de Vriese \& J.E. Teijsmann s.n. (lectotype L [L0057497], designated here).

Lasianthus polycarpus Miq., Ann. Mus. Bot. Lugduno-Batavi 4: 246 (1869). - TYPE: Borneo, 1857-1861, G.W. de Vriese s.n. (lectotype L [L0057500], designated here).

Lasianthus griffithii Wight var. latibracteatus King \& Gamble, J. Asiat. Soc. Bengal, Pt. 2, Nat. Hist. 73: 112 (1904), as 'latibracteata'. - TYPE: [Peninsular Malaysia], Selangor, Petaling, 1899, H.N. Ridley 10196 (lectotype CAL [CAL0000031878], designated here).

Tan et al. (2008) assessed this species as presumed nationally extinct in Singapore. However, there have been a couple of recent collections of Lasianthus griffithii from Nee Soon (W.F. Ang et al. s.n., 25 Mar 2011, SINU; L.M.J. Chen et al. SING2017-671, 28 Nov 2017, SING). A recent visit confirmed that at least one individual is still present (Fig. 1).

The Singapore specimens of Lasianthus griffithii are notable for being almost entirely glabrous. A collection made by Ridley at Mt Austin in Johore is similar. Otherwise the specimens conform to Lasianthus griffithii and I therefore see no reason to make any formal taxonomic distinction for the glabrous plants.

6. Lasianthus hirsutus (Roxb.) Merr., J. Arnold Arbor. 33: 229 (1952). - Triosteum hirsutum Roxb., Fl. Ind. 2: 180 (1824). - Lasianthus roxburghii Wight, Calcutta J. Nat. Hist. 6: 502 (1846), nom. illeg. (superfl). - TYPE: [Bangladesh], Chittagong, Anon. sp. no. 139 (lectotype BM [BM000848507], first step designated by Merrill, J. Arnold Arbor. 33: 230 (1952), second step designated here). 

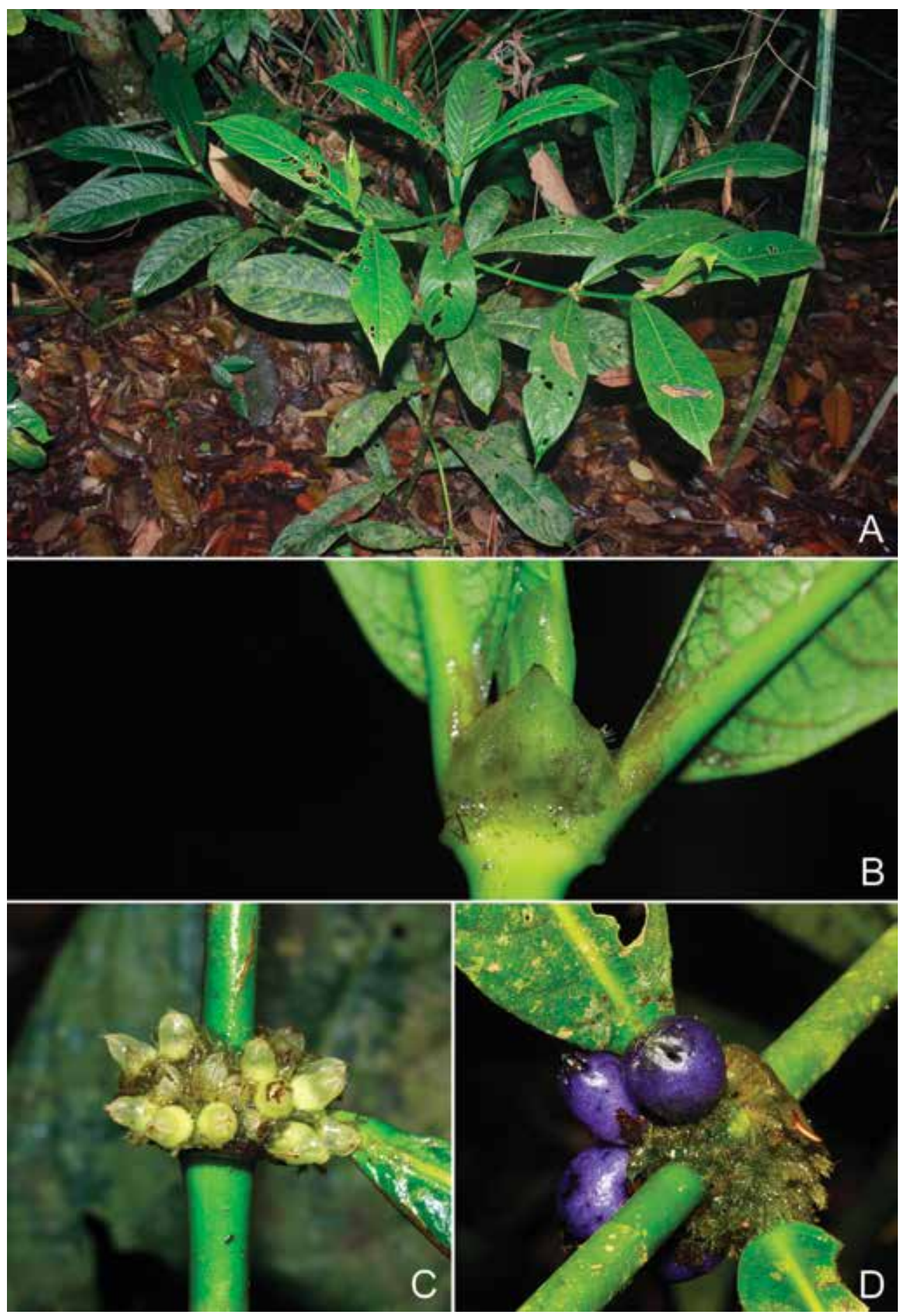

Fig. 1. Lasianthus griffithii Wight growing at Nee Soon. A. Habit. B. Large stipule. C. Inflorescence. D. Infructescence. All from SING 2017-671. (Photos: L.M.J. Chen). 
Lasianthus bracteatus Wight, Calcutta J. Nat. Hist. 6: 501 (1846), as 'bractiatus'. - Mephitidia bracteata (Wight) Walp., Ann. Bot. Syst. 2: 759 (1852). - TYPE: [Peninsular Malaysia], Malacca, W. Griffith s.n. (not traced).

Lasianthus oculus-cati Miq., Fl. Ned. Ind. 2: 315 (1857), as 'oculus cati'. - TYPE: Java, Tjidoek Tjigalin, 4 September 1842, H. Zollinger 593 (lectotype P [P04005337], designated here; isolectotypes BM, P [P04005347]).

Lasianthus laevicaulis Kurz, J. Bot. 13: 327 (1875). - TYPE: Nicobar Islands, Kamorta, February 1875, W. Kurz s.n. (lectotype K [K000031667], first step designated by Deb \& Gangopadhyay, J. Econ. Taxon. Bot. 15: 282 (1991), second step designated here; possible isolectotypes CAL, K).

Lasianthus everettii Merr., Philipp. J. Sci., C 3: 265 (1908). - TYPE: Philippines, Negros Occidental, Everett Forest Bur. 5591 (not traced).

Lasianthus cyanocarpus var. novaguineensis Valeton, Nova Guinea 8: 498 (1911). - TYPE: SW New Guinea, Noord-Fluss, umgegend des Geluks-Hügels, Versteeg 1406 (lectotype L [L0310256], designated here; isolectotype U [U0227184]).

Lasianthus cyanocarpus var. asperatus Pierre ex Pit. in Lecomte, Fl. Indo-Chine 3: 381 (1924). - TYPE: Vietnam, Cochinchine, prov. Bienhoa, ad Gia Lau Mé, September 1869, L. Pierre 3256a (lectotype P [P03980331], designated here, isolectotypes P [P03980332, P03980336]).

Lasianthus cyanocarpus var. lucidulus Pierre ex Pit. in Lecomte, Fl. Indo-Chine 3: 382 (1924). - TYPE: Vietnam, Cochinchine, pref. Bioen Hoa, ad Gia Lau Mé, September 1865, L. Pierre $3256 b$ (lectotype P [P03980334], designated here; isolectotype L).

Lasianthus cyanocarpus var. bracteatus Pit. in Lecomte, F1. Indo-Chine 3: 382 (1924). - TYPE: Vietnam, Cochinchine, Gi Tinh, 1862-1866, Thorel 1464 (lectotype $\mathrm{P}$ [P03980340], designated here; isolectotype P [P03980342]).

Lasianthus cyanocarpus auct., non Jack, King \& Gamble, J. Asiat. Soc. Bengal, Pt. 2, Nat. Hist. 73: 113 (1904); Ridley, J. Straits Branch Roy. Asiat. Soc. 33: 99 (1900); Ridley, Fl. Malay Penins. 2: 153 (1923), p.p.; Wong, Tree Fl. Malaya 4: 370 (1989) 370, p.p.; Keng, Concise Fl. Singapore, vol. 1, Gymn. Dicot. 157 (1990); Turner, Gard. Bull. Singapore 45(1): 199 (1993); Turner, Gard. Bull. Singapore 47(2): 430 (1997 ['1995']); Chong et al., Checkl. Fl. Singapore 175 (2009).

Merrill (1952) referred to two sheets in BM as the type of Triosteum hirsutum Roxb. but there is no indication on them that they are to be treated as a single specimen, though they do appear to be true duplicates. The specimen with the annotation of Roxburgh's name in Roxburgh's hand is here chosen as lectotype by a second-step designation. 
L. hirsutus.

The name Lasianthus cyanocarpus has frequently been misapplied to

7. Lasianthus reticulatus Blume, Bijdr. Fl. Ned. Ind. 1000 (1826). - Nonatelia reticulata (Blume) Kuntze, Revis. Gen. P1. 1 (1891) 291. - TYPE: Java, Gunong Seribú, C.L. Blume 862 (lectotype L [L0000700], designated here).

Lasianthus cinnamomoides Miq., Fl. Ned. Ind. 2: 325 (1857). - Nonatelia cinnamomoides (Miq.) Kuntze, Revis. Gen. P1. 1: 291 (1891). - TYPE: Java, Passir Madang, 12 August 1843, H. Zollinger 1478 (lectotype P [P04008190], designated here; isolectotypes P [P04008191, P04008194, P04008192]).

Lasianthus maingayi Hook.f., Fl. Brit. India 3: 188 (1880). - Nonatelia maingayi (Hook.f.) Kuntze, Revis. Gen. P1. 1: 291 (1891). - TYPE: [Peninsular Malaysia], Malacca, 13 April 1867, A.C. Maingay 1699 [Kew distrib. no. 871] (lectotype K [K000763918, K000763917 - i.e. a single specimen over two sheets], designated here).

Lasianthus caloneurus K.Schum., Bot. Tidsskr. 24: 339 (1902). - TYPE: Thailand, Koh Chang, Klong Son, 2 March 1900, J. Schmidt 683 (holotype C [C10018243]).

Lasianthus flavicans King \& Gamble, J. Asiat. Soc. Bengal, Pt. 2, Nat. Hist. 73: 116 (1904). - TYPE: [Peninsular Malaysia], Perak, September 1886, King's Collector 10974 (lectotype CAL [CAL0000031871], designated here).

Lasianthus flavicans var. subglaber King, J. Asiat. Soc. Bengal, Pt. 2, Nat. Hist. 73: 116 (1904). - Lasianthus maingayi var. subglaber (King) Ridl., Fl. Malay Penins. 2: 160 (1923), as 'subglabra'. - TYPE: [Peninsular Malaysia], Selangor, Langat, 1 July 1889, H.N. Ridley 4895 (lectotype CAL [CAL0000031870], designated here; isolectotype $\mathrm{K}$ [K001129563]).

8. Lasianthus ridleyi King \& Gamble, J. Asiat. Soc. Bengal, Pt. 2, Nat. Hist. 73: 115 (1904). - TYPE: Singapore, Bukit Mandai, 1892, H.N. Ridley 3620 a (lectotype CAL [CAL0000031868], designated here, or possibly holotype).

Lasianthus reticulatus var. polydasys Miq., Ann. Mus. Bot. Lugduno-Batavi 4: 248 (1869). - TYPE: Sumatra, P.W. Korthals s.n. (lectotype L [L0305485], designated here; possible isolectotype L [L0305487]).

Lasianthus singaporensis King \& Gamble, J. Asiat. Soc. Bengal, Pt. 2, Nat. Hist. 73: 117 (1904). - TYPE: Singapore, Bukit Timah, 19 September 1897, H.N. Ridley 9095 (lectotype SING [SING0059268], designated here; isolectotype CAL (CAL000031866)). 
Lasianthus maingayi var. hirtus Ridl., Fl. Malay Penins. 2: 160 (1923), as 'hirta'. - TYPE: [Peninsular Malaysia], Negri Sembilan, Bukit Tangga, December 1920, H.N. Ridley s.n. (lectotype K [K000763939], designated here; isolectotype SING [SING0059761]).

9. Lasianthus scabridus King \& Gamble, J. Asiat. Soc. Bengal, Pt. 2, Nat. Hist. 73: 111 (1904). - Lasianthus griffithii var. scabridus (King \& Gamble) H.Zhu in Zhu et al., Blumea 57: 46 (2012). - TYPE: [Peninsular Malaysia], Johor, Tanjong Bunga, 1894, H.N. Ridley 6463 (lectotype SING [SING0059269], designated by Wong, Arbor. Rubiaceae Malaya 113 (1988); isolectotype K [K000763943]).

Zhu (Zhu et al., 2012) reduced Lasianthus scabridus to a variety of L. griffithii. The affinity of the two taxa is clear, but Lasianthus scabridus is very consistent morphologically and quite readily distinguished from L. griffithii by a number of characters. Lasianthus scabridus has leaves scabrid above, lateral nerves to 12 pairs, petiole generally extending beyond the inflorescence with scattered long hairs visible to the naked eye whereas L. griffithii has leaves smooth above, lateral nerves 14 or more pairs, petiole largely hidden by the inflorescence, without long hairs visible to the naked eye. I therefore prefer to maintain recognition at species rank for this taxon.

10. Lasianthus stipularis Blume, Bijdr. Fl. Ned. Ind. 997 (1826). - Mephitidia stipularis (Blume) DC., Prodr. 4: 453 (1830). - Nonatelia stipularis (Blume) Kuntze, Revis. Gen. P1. 1: 291 (1891). - TYPE: Java, Salak, C.L. Blume 674 (lectotype L [L0000708], designated here; isolectotype L [L0000709]).

Lasianthus stipularis var. novaguineensis Valeton, Nova Guinea 8: 498 (1911). - TYPE: New Guinea, [Indonesia], fluv. Noordrivier, G.M. Versteeg 1228 (lectotype L [L0305625, L0305626, L0305627 - i.e. a single specimen over three sheets], designated here; isolectotypes K [K000777085, K000777084], U [U0247139]).

11. Lasianthus venosus Blume, Bijdr. Fl. Ned. Ind. 990 (1826). - Mephitidia venosa (Blume) DC., Prodr. 4: 453 (1830). - Nonatelia venosa (Blume) Kuntze, Revis. Gen. P1. 1: 291 (1891). - TYPE: Java, C.L. Blume 887 (lectotype L [L0000711], designated here).

Lasianthus pterospermus Wight, Calcutta J. Nat. Hist. 6: 510 (1846). - Mephitidia pterosperma (Wight) Walp., Ann. Bot. Syst. 2: 762 (1852). - Nonatelia pterosperma (Wight) Kuntze, Revis. Gen. P1. 1: 291 (1891). - TYPE: [Peninsular Malaysia], Malacca, W. Griffith s.n. (holotype K [K000763920], fide Noltie, Bot. Robert Wight 416 (2005)). 
Lasianthus singalensis Miq., Ann. Mus. Bot. Lugduno-Batavi 4: 247 (1869). - TYPE: Sumatra, P.W. Korthals s.n. (lectotype L [L0057495], designated here; possible isolectotype L [L0057496]).

\section{Mussaenda}

The generic name Landia was published by Adrien de Jussieu based on material collected on Mauritius by Commerson. Poiret later published two species names, Mussaenda landia Poir. and M. arcuata Poir., from the Commerson collections. The generic name Landia Comm. ex Juss. seems not to have been typified although the choice of type is between Mussaenda landia and M. arcuata (viz. ICN Art. 10.2). The former is the type of Bremeria Razafim. \& Alejandro, a genus from the Indian Ocean (Madagascar and Mascarenes) that was recently separated from Mussaenda (Alejandro et al., 2005). The latter belongs in Mussaenda sensu stricto (Alejandro et al., 2005). Alejandro et al. (2005) cited Landia as a synonym of Mussaenda s.s. This makes the selection of Mussaenda arcuata as the neotype for Landia a nomenclaturally stabilising step, and is done below, though as Landia is a later homonym, this is of purely academic significance. De Candolle described Mussaenda section Landia including reference to Commerson's herbarium name, but not Jussieu's publication. He included both Mussaenda landia and M. arcuata in the section, as well as three other species. It seems convenient to select Mussaenda arcuata as the lectotype of de Candolle's section also so that the generic and sectional names are homotypic. It also allows the possibility of using the sectional name in an infrageneric classification of Mussaenda s.s.

Landia Comm. ex Juss., Gen. Pl. 201 (1789), nom. illeg., non Dombey (1784). - TYPE: Mussaenda arcuata Poir. (neotype designated here)

Mussaenda sect. Landia Comm. ex DC., Prodr. 4: 372 (1830). - TYPE: Mussaenda arcuata Poir. (lectotype designated here)

Singapore only has two native species of Mussaenda. The names and synonymies are given below as some lectotypifications are required.

1. Mussaenda glabra Vahl, Symb. Bot. 3: 38 (1794). - Mussaenda frondosa var. glabra (Vahl) Miq., Fl. Ned. Ind. 2: 213 (1857). - TYPE: Anon. s.n. (lectotype C [C10018260], designated by Jayaweera, J. Arnold Arbor. 44: 265 (1963)).

Mussaenda penangensis Miq., Fl. Ned. Ind. 2: 214 (1857). - TYPE: [Peninsular Malaysia], Penang, N. Wallich s.n. [EIC 6251A] (lectotype L [L0843026], designated here). 
Mussaenda glabra var. puberula King, J. Asiat. Soc. Bengal, Pt. 2, Nat. Hist. 72: 186 (1904). - TYPE: Singapore, 1859, R. Schomburgk 43 (lectotype K [K001129410], designated by Chantaranothai, Thai Forest Bull., Bot. 43: 55 (2015)).

Mussaenda andersonii Soumen K.Basu \& T.K.Paul, J. Bombay Nat. Hist. 86: 430 (1990 ['1989']). - TYPE: India, Sikkim, Kolwong, 9 May 1862, T. Anderson 163 (holotype CAL).

2. Mussaenda maingayi (Hook.f.) Hemsl. ex T.Durand \& B.D.Jacks., Index Kew., Suppl. 1: 284 (1903). - Acranthera maingayi Hook.f., Fl. Brit. India 3: 92 (1880). - Asemanthia maingayi (Hook.f.) Ridl., Bull. Misc. Inform. Kew 1939: 600 (1940). - TYPE: [Peninsular Malaysia], Malacca, 16 July 1867-1868, A.C. Maingay 2449 [Kew distrib. no. 940] (lectotype K [K000740953], designated here).

Acranthera griffithii Hook.f., Fl. Brit. India 3: 92 (1880). - Mussaenda griffithii (Hook.f.) Hemsl. ex B.D.Jacks., Index Kew., Suppl. 1: 284 (1903). - Asemanthia griffithii (Hook.f.) Ridl., Bull. Misc. Inform. Kew 1939: 600 (1940). - TYPE: East Himalaya, W. Griffith s.n. [Kew distrib. no. 3089] (lectotype L [L0000132], designated here; possibly isolectotypes C [2 sheets], GH, L).

Acranthera mutabilis Hemsl., J. Bot. 25: 204 (Jul 1887). - Mussaenda mutabilis (Hemsl.) Hemsl., Hooker's Icon. P1. 18: t, 1718 (Nov 1887). - TYPE: [Peninsular Malaysia], Perak, Waterfall Hill, L. Wray 91 (holotype K [K001129440]; isotype SING [SING0062184]).

Mussaenda mutabilis var. hirsuta King, J. Asiat. Soc. Bengal, Pt. 2, Nat. Hist. 72: 182 (1904). - Asemanthia maingayi var. hirsuta (King) A.K.Sinha \& B.Mitra, J. Bombay Nat. Hist. Soc. 78: 427 (1981). - TYPE: [Peninsular Malaysia], Johore, Gunong Panti, June 1880, King's Collector 225 (lectotype K [K0007409549], designated here; isolectotypes CAL, K [K0007409548]).

Mussaenda mutabilis var. montana Ridl., Fl. Malay Penins. 2: 58 (1923). - Asemanthia maingayi var. montana (Ridl.) A.K.Sinha \& B.Mitra, J. Bombay Nat. Hist. Soc. 78: 428 (1981). - TYPE: [Peninsular Malaysia], Malacca, Mount Ophir, Padang Batu, H.N. Ridley 3215 (lectotype CAL, designated by Sinha \& Mitra, J. Bombay Nat. Hist. Soc. 78: 428 (1981); isolectotype SING [SING0240244]).

Original material of Acranthera griffithii would be expected in $\mathrm{K}$, but I have failed to locate any. I therefore select the good-quality sheet in L as lectotype here. The locality of East Himalaya indicated on the Kew distribution ticket seems likely to be an error. 


\section{Psychotria}

The generic name Uragoga Baill. is widely cited as a synonym of Psychotria L., with its place of publication generally being given as page 323 in volume 12 of Henri Baillon's journal Adansonia. This is in a paper entitled 'Memoire sur les Uragoga'. Baillon published Adansonia in parts of 32 pages with 12 parts completing a volume. The early volumes had parts published in consecutive months, but the output became more erratic in later volumes. For volume 12, publication dates are printed on the first page of each 32-page part. The memoire on Uragoga appeared in part 11 published in August 1879. However, while this paper provided an overview of Baillon's generic concept of Uragoga, he had already described some 41 species, very largely from New Caledonia, in the genus Uragoga in preceding parts of Adansonia volume 12. This is nomenclaturally problematic because if the generic name Uragoga was not validated until part 11, the binonials in Uragoga published before the validation of the generic name would be invalid. It would then be necessary to search for later validations of these species names which could lead to changes in author citations or even to names in current use.

However, there does seem to be a possibility of avoiding this problem. In the discussionary notes placed after the description of the first species of Uragoga published by Baillon (p. 223), the following Latin sentence appears: Stirps inde, uti americanae asiaticaeque nonnullae, arctius Psychotriam cum Cephaelide (i.e. Uragoga L.) connectit.

This can be translated as: Thence this plant [i.e. Uragoga pancheri Baill.], just like certain American and Asiatic ones, joins Psychotria more closely with Cephaelis (i.e. Uragoga L.).

The interpretation that Baillon was here proposing Uragoga (a name used but not validated by Linnaeus) as the name of an inclusive genus is supported by the Memoire sur les Uragoga where he recognised 42 sections in the genus, many of which were based on established generic names including Cephaelis and Psychotria. It seems likely that his view on the taxonomy of this group of the Rubiaceae was already confirmed in November 1878 when Uragoga pancheri was published. Uragoga Baill. is therefore validated on p. 223 by citation of Psychotria L. and Cephaelis Sw. as synonyms, but the name Uragoga is illegitimate, as the synonyms are legitimate names, and to be automatically typified by the type of the oldest legitimate synonym, which is Psychotria.

Establishing that Uragoga Baill. is an illegitimate and superfluous renaming of Psychotria dating from 1878 means that the 41 species names and various sectional names published by Baillon in Uragoga before August 1879 are legitimate (ICN Art. 55 ) and the currently used names for these taxa do not need to change in any way.

Psychotria L., Syst. Nat., ed. 10, 929 (1759), nom. cons. - Uragoga Baill., Adansonia 12: 223 (1878), nom. illeg. (superfl.). Type: Psychotria asiatica L.

A few notes on various Psychotria species native to Singapore are included here. 
1. Psychotria angulata Korth., Ned. Kruidk. Arch. 2(2): 243 (1851). - Uragoga angulata (Korth.) Kuntze, Revis. Gen. P1. 2: 959 (1891). - TYPE: Borneo, P.W. Korthals s.n. (lectotype L [L0843037], designated here).

Chassalia perforata Miq., Fl. Ned. Ind., Eerste Bijv. 546 (1861). - Uragoga angulosa Kuntze, Revis. Gen. P1. 1: 299 (1891). - TYPE: Bangka, J.E. Teijsmann s.n. (lectotype L [L0484307], designated here).

Psychotria griffithii var. angustifolia Ridl., Fl. Malay Penins. 2: 137 (1923). - TYPE: [Peninsular Malaysia], Lankawi, Burau Woods, April 1911, H.N. Ridley 15823 (lectotype K [K001067640], designated by Turner et al., Gard. Bull. Singapore 70: 370 (2018)).

2. Psychotria griffithii Hook.f., Fl. Brit. India 3: 171 (1880). - Uragoga malaccensis Kuntze, Revis. Gen. P1. 2: 956 (1891). - TYPE: [Peninsular Malaysia], Malacca, Ayer Panas, W. Griffith s.n. [Kew distrib. no. 3044] (lectotype K [K000777141], designated here).

3. Psychotria morindiflora Wall. ex Hook.f., Fl. Brit. India 3: 166 (1880), as 'morindaeflora'. - Uragoga morindiflora (Wall. ex Hook.f.) Kuntze, Revis. Gen. P1. 2 (1891) 961. - TYPE: Singapore, October 1822, N. Wallich s.n. [EIC 8438A] (lectotype K-W [K001125515], designated by Turner \& Kumar, Phytotaxa 361: 189 (2018)).

Turner \& Kumar (2018) confirmed that this species should be considered native to Singapore but they cited no specimens collected after 1908. I recently found two more recent collections from Nee Soon in the herbarium of the Lee Kong Chian Natural History Museum (H.T.W. Tan 2/7.5.82-1, 7 May 1982, SINU; P.P.Y. Lim et al. NRS 770; 6 May 1992, SINU). This surely raises the possibility that this climbing Psychotria species is still extant in Singapore.

ACKNOWLEDGEMENTS. I am very grateful to V. Sampath Kumar (CAL) for information on type material in the Central National Herbarium, Mats Hjertson (UPS) for supplying an image of the Lasianthus brachyphyllus type, Melanie Thomas (K) for Latin translation, John McNeill (E) for lengthy correspondence on the nomenclature of Uragoga, and Chua Keng Soon (SINU) for assistance during a visit to LKCNHM.

\section{References}

Alejandro, G.D., Razafimandimbison, S.G. \& Liede-Schumann, S. (2005). Polyphyly of Mussaenda inferred from ITS and trnT-F data and its implication for generic limits in Mussaendeae (Rubiaceae). Amer. J. Bot. 92: 544-557. 
Ali, S.J. \& Robbrecht, E. (1991). Remarks on the tropical Asian and Australian taxa included in Diplospora or Tricalysia (Rubiaceae - Ixoroideae - Gardenieae). Blumea 35: 279-305. Arriola, A.H., Davis, A.P., Davies, N.M.J., Meve, U., Liede-Schumann, S. \& Alejandro, G.J.D. (2018). Using multiple plastid DNA regions to construct the first phylogenetic tree for Asian genera of Coffeeae (Ixoroideae, Rubiaceae). Bot. J. Linn. Soc. 188: 132-143.

Hooker, J.D. (1880). The Flora of British India, vol. 3, pt. 7. London: L. Reeve \& Co.

Merrill, E.D. (1952). William Jack's genera and species of Malaysian plants. J. Arnold Arbor. 33: 199-251.

Tan, H.T.W., Tan, K.-X., Ibrahim, A., Chew, P.T., Chua, K.S., Duistermaat, H., Ganesan, S.K., Goh, M.W.K., Gwee, A.T., Kiew, R., Lee, S.M.L., Leong, P., Lim, J., Lok, A.F.S.L., Loo, A.H.B., Lum, S.K.Y., Morgany, T., Suran, S., Sim, S., Ahmad, S., Wee, Y.C., Yap, K.F., Yeo, C.K. \& Yong, J.W.H. (2008). Seed plants. In: Davison, G.W.H., Ng, P.K.L. \& Ho, H.C. (eds) The Singapore Red Data Book: Threatened Plants and Animals of Singapore, $2^{\text {nd }}$ ed., pp. 213-245. Singapore: Nature Society (Singapore).

Turland, N.J., Wiersema, J.H., Barrie, F.R., Greuter, W., Hawksworth, D.L., Herendeen, P.S., Knapp, S., Kusber, W.H., Li, D.Z., Marhold, K., May, T.W., McNeill, J., Monro, A.M., Prado, J., Price, M.J. \& Smith, G.F. (eds) (2018). International Code of Nomenclature for algae, fungi, and plants (Shenzhen Code) adopted by the Nineteenth International Botanical Congress Shenzhen, China, July 2017. Regnum Vegetabile 159. Glashütten: Koeltz Botanical Books.

Turner, I.M. \& Kumar, V.S. (2018). Flora of Singapore precursors, 4. A summary of scandent Psychotria (Rubiaceae) in Singapore and Peninsular Malaysia. Phytotaxa 361: 183-197.

Wong, K.M. (1989). Rubiaceae. In: Ng, F.S.P. (ed.) Tree Flora of Malaya, vol. 4, pp. 324-425. Petaling Jaya: Longman Malaysia Sdn. Berhad.

Zhu, H., Roos, M.C. \& Ridsdale, C.E. (2012). A taxonomic revision of the Malesian species of Lasianthus (Rubiaceae). Blumea 57: 1-102. 